\title{
Die Rolle der Medien in der COVID-19-Pandemie
}

\author{
Erste inhaltsanalytische Befunde
}

\begin{abstract}
Georg Ruhrmann ${ }^{(凶)}$ und Dominik Daube
Institut für Kommunikationswissenschaft, Friedrich-Schiller-Universität Jena, Jena, Deutschland

\{Georg.Ruhrmann, dominik.daube $\}$ duni-jena.de
\end{abstract}

Schlüsselwörter: Öffentlichkeit · Soziale Medien ·

Wissenschaftskommunikation

Der vorliegende Text ist eine überarbeitete und erweiterte Fassung des Vortrages, den Prof. Dr. Georg Ruhrmann am 30. Oktober 2020 im Rahmen der Tagung „Infektionen und Gesellschaft" gehalten hat.

\section{Einleitung}

Presse, Rundfunk und Social Web präsentieren seit Monaten dramatische Schlagzeilen, Texte und Titel. Es gibt zeitweilig seit März 2020 keine anderen Themen mehr. ${ }^{1}$ Wir werden medial mit verschiedenartigsten Folgen von COVID-19 konfrontiert. Was kann Sozialwissenschaft angesichts von ,so viel Wissen über unser Nichtwissen ... und dem Zwang, unter Unsicherheit leben und handeln zu müssen“ [2] schon „sehen“ und „deuten“ [3]? Was ist die Rolle der Medien? Wie berichten Medien von der laufenden Pandemie? Und: Wie lässt sich diese Berichterstattung systematisch analysieren [vgl. 4]? Damit verbunden sind zwei klassische Fragen: Dramatisieren die Medien eine ohnehin schon dramatische Lage zusätzlich? Oder wie ausgewogen repräsentieren sie die Akteurinnen und Akteure, Ereignisse und Entwicklungen [vgl. 5-7]? Diese Fragen wurden bereits bei früheren Pandemien, etwa der Schweinegrippe (H1N1) [vgl. 8], und der Ebola-Krise 2014 [vgl. 9], untersucht.

Wir wissen aus empirischen Studien, dass Journalistinnen und Journalisten je nach Einstellungen und Vorwissen sowie nach Anlass und Ereignis spezifisch und unterschiedlich über gesundheitliche und wissenschaftliche Themen im Allgemeinen [1012] berichten. Dies gilt speziell auch für Pandemien [8, 13-15].

1 Bis zu $60 \%$ aller Online-Artikel (450 von 750) der Journalistinnen und Journalisten des SPIEGEL nahmen in der 13. Kalenderwoche (23.-29.03.20) Bezug zu COVID-19 [1]. 
Diese sozialwissenschaftlichen Erkenntnisse zur Rolle der Medien, die auf empirischen Untersuchungen basieren, sind häufig nicht bekannt, gerade in der organisierten Politikberatung zu medizinischen Fragen und Themen fehlen häufig die Kenntnisse. $^{2}$

Was zeigen erste Analysen und Reviews zu systematischen Inhaltsanalysen der Medienberichterstattung zur Pandemie? „Results show that media attention was immense, that news content stressed threat over precautionary measures, while the pattern of coverage tonality remained nebulous due to conflicting findings " [21]. Eine Metaanalyse von $n=118$ Studien zum gesamten Kommunikationsprozess stellt fest: „Trust in public officials and source of information, worry and levels of knowledge about the di-sease, and routine media exposure as well as information-seeking behaviors, were related to greater likelihood of adoption of recommended infection prevention practices“ [22]. Weitere vergleichende Analysen zur Kommunikation über Infektionskrankheiten zeigen, welche Aspekte der Pandemiebekämpfung in Öffentlichkeiten des Social Web wichtig waren und vermutlich wichtig werden [23]. Zudem lässt sich schon ansatzweise die geänderte Rolle und die „cultural authority“ [24] der Wissenschaft während des Verlaufs der Pandemie beobachten.

\section{Ziel und Fragestellung}

Weil die Erstellung und Publikation komplexer Analysen - wie schon angemerkt einige Zeit benötigen, ist jetzt und in naher Zukunft mit weiteren empirischen Studien zu rechnen. Der nachfolgende Artikel verfolgt das Ziel, bisherige systematische Medieninhaltsanalysen zu erörtern, und zwar bezogen auf die Fragen:

1. Wie wird in den Inhaltsanalysen über die medial dargestellten wissenschaftliche Expertinnen und Experten, Medizinerinnen und Mediziner und ihre Themen in der der laufenden Pandemie berichtet?

2. Wie werden in den Studien relevante rechtliche und politische Akteurinnen und Akteure und ihre Entscheidungen dargestellt?

3. Wie werden in den inhaltsanalytischen Untersuchungen mögliche Reaktionen und Verhaltensweisen einzelner Bevölkerungsgruppen präsentiert?

4. Wie wird in den bisher vorliegenden Studien Evidenz des wissenschaftlichen Wissens präsentiert?

5. Wie analysieren vorhandene Untersuchungen die Darstellung beziehungsweise Repräsentanz von Desinformationen und Verschwörungstheorien zur COVID-19Pandemie?

Der Artikel kann nicht beanspruchen, dazu schon eine Art systematischer Inhaltsanalyse von sozialwissenschaftlichen Inhaltsanalysen oder gar eine Meta-Analyse bisheriger Inhaltsanalysen zu COVID-19 vorzunehmen. Das bleibt einer späteren Untersuchung vorbehalten [25].

2 Deren öffentliche Wirkungen können - wie nun sichtbar wird - komplexer sein als in einer nur auf ,best practice“ ausgerichteten Wissenschaftskommunikation [14-20]. 


\section{Inhaltsanalysen zu COVID-19}

Erste Studien geben einen ersten Eindruck, wie die bisherige Berichterstattung während früherer Pandemien und vor allem der aktuellen COVID-19 Pandemie ablief. Die Berichterstattung in dynamischen Pandemiesituationen wurden in der Vergangenheit bereits im Kontext von H1N1 [21] oder MERS [26] untersucht. Aktuell kann die Berichterstattung über COVID-19 aufklären und ein besseres Verständnis für die Evolution der Pandemie, der damit einhergehenden sozialen Krise [vgl. 27] und für gesundheitspolitische Interventionsmaßnahmen schaffen.

Gleichzeitig bergen insbesondere Desinformationen zur Pandemie, vor allem in den sozialen Medien [vgl. 28], auch Risiken von Panikverbreitung [19] oder Stress $[29$, 30]. Relativ unstrittig ist dabei allerdings - wie schon erwähnt - die Aufklärungsleistung der klassischen journalistischen Medien und auch ihr Beitrag zur Krisenkommunikation [vgl. 31]. Derzeit werden über Live-Ticker und eine tagesaktuelle - häufig sogar minutenaktuelle - Berichterstattung die Hygiene- und weiteren Präventionsmaßnahmen reichweitenstark kommuniziert. Damit dienen die klassischen Medien auch als ein wichtiges Sprachrohr der Wissenschaftlerinnen und Wissenschaftler an die Öffentlichkeit [29].

Aktuell analysieren unterschiedliche Wissenschaftsdisziplinen die Berichterstattung und ihre Auswirkungen in vielfältigen Kontexten. Stellvertretend kann an dieser Stelle die Tourismus-Branche genannt werden, die stark von der Pandemie betroffen ist. Daher wurden die Auswirkungen der Berichterstattung auf das Reiseverhalten untersucht [32]. Die mediale Krisenkommunikation wurde bereits in kurzer Zeit weltweit analysiert. Es lassen sich dadurch zumindest erste analytische und normative Überlegungen zu einer veränderten und sich verändernden Berichterstattung ableiten.

Der Artikel beleuchtet nachfolgend zunächst das Thema medial repräsentierter Wissenschaft und Medizin (Abschn. 3.1) und widmet sich dann der Darstellung von politischen und juristischen Akteuren (vgl. Abschn. 3.2) sowie Reaktionen und Verhaltensweisen einzelner Gruppen (vgl. Abschn. 3.3). Danach wird analysiert, ob und wie Medien die wissenschaftliche Evidenz berichteter Studien darstellen (vgl. Abschn. 3.4) und wie sie mit Desinformationen umgehen (vgl. Abschn. 3.5). Es folgen kurze Schlussbemerkungen (vgl. Abschn. 3.6).

\subsection{Wissenschaft und Medizin}

Zunächst wird die mediale Repräsentation von Wissenschaft - konkret auch der wissenschaftlichen Disziplin Medizin - betrachtet. Dabei soll auch auf mögliche Rollenkonstruktionen und Rollenzuweisungen von Wissenschaftlerinnen und Wissenschaftlern eingegangen werden. Konkret lassen sich die Fragen stellen, wie die Wissenschaft medial dargestellt wird, welche Akteurinnen und Akteure zu Wort kommen und wie wissenschaftliche Fakten und Informationen in den Massenmedien aufbereitet und verbreitet werden.

Bereits jetzt, mitten in der Pandemie, behandeln zahlreiche Studien diese Thematik. Ein Grund für dieses hohe Interesse: In der hochdynamischen 
Pandemiesituation wird die Wissenschaft als eine zentrale und vor allem glaubwürdige Quelle herangezogen. Und zwar mit dem Ziel, fundierte und möglichst ${ }^{3}$ korrekte Informationen zum Virus selbst, zu Präventivmaßnahmen und zur Ein- und Abschätzung möglicher Folgen für Gesellschaft und Wirtschaft zu erhalten.

Die vorhandene wissenschaftliche Unsicherheit bezüglich COVID-19 erschwert die Kommunikation über Virus und Krankheit. Eine riskante Politisierung der Thematik kann eine Folge sein. So ist die Frage nach Art und Weise der Kommunikation ebendieser Unsicherheit wichtig, um riskante Missverständnissen effektiv vorbeugen zu können. Wie könnte eine Strategie hier aussehen und gibt es Anhaltspunkte, wie eine erfolgreiche Wissenschaftskommunikation aussehen sollte?

Kreps und Kriner nahmen sich dieser Thematik an und zeigten unter anderem, dass eine vorneherein starke Betonung bestehender Unsicherheiten (vgl. Abschn. 3.5) einen negativen Einfluss auf das Vertrauen der Öffentlichkeit haben kann [33]. Spielt man jedoch die Unsicherheit herunter oder kommuniziert sie gar nicht, so ist kurzfristig zwar mit erhöhter Akzeptanz und Unterstützung der kommunizierten Maßnahmen zu rechnen. Längerfristig betrachtet birgt die Strategie jedoch die Gefahr einer Abschwächung der Glaubwürdigkeit, bis hin zum nachhaltigen und gegebenenfalls irreversiblen Verlust des Vertrauens in die Wissenschaft [33].

Dies verdeutlicht, wie wichtig eine sorgfältige und überlegte Wissenschaftskommunikation ist, um sicherzustellen, dass die Öffentlichkeit die pandemiebezogenen Maßnahmen und deren Notwendigkeit nachvollziehen kann und bereit ist, diese mitzutragen und zu unterstützen. Dies erscheint umso wichtiger, wenn die politischen Entscheidungen auf wissenschaftlichen Erkenntnissen basieren und hiervon maßgeblich beeinflusst werden.

Gerade zu Beginn der Pandemie war die wissenschaftliche Unsicherheit besonders hoch. Journalistinnen und Journalisten haben im Normalfall den Anspruch, glaubhafte und qualitativ hochwertige Informationen aus der Wissenschaft zu erhalten. Nun mussten sie - entgegen den regulären Recherchepraktiken und Recherchemaßstäben gezwungenermaßen mit diesen unsicheren Erkenntnissen arbeiten, sie aufbereiten und für Laien verständlich kommunizieren, um die neuartige Erkrankung überhaupt in die Berichterstattung aufnehmen zu können [vgl. 34].

Ein zügiges Prüfen, Aufbereiten und Weitergeben der immer wieder neuen, aktuelleren Informationen und somit eine kontinuierliche Informationsverbreitung an Journalistinnen und Journalisten wird vom Science Media Center in Deutschland praktiziert. Dieses stellt eine Schnittstelle der Informationsvermittlung zwischen Wissenschaft und Journalismus dar [35, 36].

Wissenschaftliche Inhalte werden gerade in der laufenden Pandemie jedoch nicht immer journalistischen Prüfungen unterzogen. Zunehmend kommunizieren Wissenschaftlerinnen und Wissenschaftler neueste Erkenntnisse über verschiedene Kanäle, wie Podcasts und Social Media. Wormer stellte im Mai 2020 fest, dass die

3 Bei einer Pandemie mit einem neuen Erreger müssen zunächst neue wissenschaftliche Erkenntnisse gesammelt werden - somit besteht vor allem zu Beginn eine hohe Unsicherheit, verbunden mit diversen weiteren Informationen und Meinungen. Deshalb wird an dieser Stelle von möglichst korrekten Informationen gesprochen. 
Grenzen zwischen Wissenschaftsjournalismus und der Wissenschaftskommunikation durch Wissenschaftlerinnen und Wissenschaftler heute zunehmend verschwimmen [37]. Er prognostiziert, dass diese Entwicklung gerade in Zeiten der Pandemie noch beschleunigt wird. Dabei nimmt er explizit Bezug auf bisherige Erkenntnisse und Stellungnahmen unter anderem der Leopoldina, welche die präzisere Unterscheidung zwischen klassischer Wissenschaftskommunikation und der PR-orientierten Öffentlichkeitskommunikation wissenschaftlicher Inhalte nahelegen. Wormer kommt unter anderem zu dem Schluss, dass wissenschaftliche Institutionen ihre Kommunikation reformieren müssen. So sei es vorzuziehen, die Öffentlichkeitsarbeit dieser Institutionen an der gezielten Information über wissenschaftliche Abläufe und Erkenntnisse auszurichten, anstatt - wie bisher - bei der Kommunikation das primäre Ziel einer verbesserten und positiveren öffentlichen Wahrnehmung der Institution zu verfolgen. Dies setzt neue Abläufe und ein interdisziplinäres Vorgehen voraus, an welchem Expertinnen und Experten der Wissenschaftskommunikation unbedingt beteiligt werden sollen.

Wie bereits angeklungen, spielen - insbesondere in der ,ungefilterten“ Kommunikation - die sozialen Medien eine Schlüsselrolle. Wie die Wissenschaftskommunikation über diese Kanäle ablaufen kann beziehungsweise welche Rolle die sozialen Medien spielen, beschreiben Pollett und Rivers [38]. So schreiben sie konkret über das soziale Medium Twitter und schreiben ihm eine fundamentale, wichtige Rolle beim synchronen Austausch von neuen wissenschaftlichen Erkenntnissen zu. Nicht nur die Politik nutzt diesen Dienst als Kommunikationskanal, sondern auch Expertinnen und Experten aus der Wissenschaft - nicht nur zur Informationsverbreitung an die Öffentlichkeit, sondern auch zum Informationsaustausch untereinander. Gleichzeitig beschreiben Pollett und Rivers diese Rolle von Twitter als „,prekär“ und zeigen die potenziellen Risiken einer - gegebenenfalls unbewussten Verbreitung von Falschinformationen - auch von Wissenschaftlerinnen und Wissenschaftlern untereinander - auf, die mit der Nutzung dieser Dienste im Allgemeinen einhergeht (vgl. Abschn. 3.5).

Murri et al. analysieren die wissenschaftliche Kommunikation über soziale Medien in der Pandemie in Italien [39]. Zur Sprache kommt die Informationssuche von praktizierenden Ärztinnen und Ärzten über Social Media anstatt über Fachzeitschriften, um hochaktuelle Informationen zu bekommen. Die Studie zeigt unter anderem einen Anstieg der Verwendung von Social Media als Informationsquelle bei Klinikerinnen und Klinikern in Italien. Soziale Medien haben einen bedeutenden Einfluss auf den täglichen klinischen Umgang mit der Erkrankung, und zwar in Bezug auf alle Altersgruppen.

Wie diese ersten Eindrücke deutlich machen, spielen die sozialen Medien nicht nur bei der Einstellungs-, Meinungs- und Wissensgenerierung von Laien und der allgemeinen Bevölkerung eine Rolle. Auch Expertinnen und Experten nutzen diese Angebote. Diese Perspektive ist für zukünftige Studien interessant, um die Thematik kritisch reflektieren und diskutieren zu können.

Nowakowska et al. zeigen eine hohe Veröffentlichungsdichte von COVID-19bezogenen Studien, unter anderem bedingt durch ein beschleunigtes Vorgehen bei der Prüfung und Veröffentlichung von COVID-19 bezogenen Studien [40]. Dies wirft 
bereits auch öffentlich diskutierte kritische Fragen nach der Belegkraft - wissenschaftliche Evidenz [15] -beziehungsweise auch nach der Relevanz der Studien auf: Wirkt sich diese schnelle Veröffentlichung positiv oder negativ auf die wissenschaftliche Evidenz und Relevanz aus [vgl. 41]? Wie beeinflusst dies die Qualität aktueller, insbesondere journalistischer Berichterstattung über ständig neue wissenschaftliche Erkenntnisse in der hochdynamischen Pandemiesituation?

\subsection{Darstellung juristischer und politischer Akteure}

Bisher gibt es erst wenige inhaltsanalytische Erkenntnisse zur Darstellung der Legislative bzw. von juristischen Akteurinnen und Akteuren.

Abbasi beschreibt in seinem Beitrag sehr kritisch den Druck, den Politik und Regierungen während der Pandemie auf die Wissenschaft ausüben [42]. Er spannt den Bogen von der Kommunikation der Wissenschaft bis hin zur Politik über diverse Kanäle. Tejedor et al. untersuchen die Titelseiten der spanischen und italienischen Tagespresse und stellen fest, dass Politikerinnen und Politiker dort am häufigsten repräsentiert werden [43]. Daraus schließen sie auf eine sichtbare Politisierung der Pandemie. Lasco betrachtet in seiner Studie einzelne politische Akteure näher und führt den Begriff des „,medizinischen Populismus“ ein [44]. Er vergleicht die COVID-19-bezogene Kommunikation der drei Staatsoberhäupter Trump, Bolsonaro und Duterte von Januar bis Juli 2020. In deren Kommunikation kristallisiert sich nach Lasco vor allem heraus, dass die Pandemie immer wieder heruntergespielt und vereinfacht dargestellt wird, ihre Bekämpfung wird außerdem mit ungeeigneten und einfachsten Maßnahmen beworben. Dabei - und hier kommt der Begriff des „medizinischen Populismus“ zum Tragen - beziehen sich die Akteure immer wieder auf vermeintliches medizinisches Wissen, welches ihre Thesen stützen soll.

Beziehen politische Führungskräfte Stellung zu COVID-19-bezogenen Maßnahmen, so zeigen sich Unterschiede in der Aufnahme dieser Kritik in der Bevölkerung hinsichtlich der politischen Ausrichtung der Akteurinnen und Akteure: Demokratische politische Eliten scheinen in der Wahrnehmung das öffentliche Vertrauen in die Wissenschaft durch geäußerte Kritik stärker zu untergraben als republikanische Eliten [33].

Nach dem Vergleich der medialen Repräsentation der politischen Ausrichtung und der Wirkung dieser auf die Berichterstattung und Meinungsbildung soll abschließend in diesem Kapitel auf die Geschlechterverteilung der medial repräsentierten Akteurinnen und Akteure eingegangen werden. Piazza und Dias nennen die generelle Annahme, dass weibliche Akteurinnen stereotypisiert sind [45]. Sie werden in der Öffentlichkeit als ehrlicher und vertrauenswürdiger wahrgenommen als männliche Akteure (auch bei Gesundheitsfragen). So kommen sie zu der Annahme, dass die verstärkte mediale Repräsentation von weiblichen Führungskräften und Politikerinnen im Allgemeinen zu einer höheren Akzeptanz von Maßnahmen führt auch weiter führen könnte. Hier führen Piazza und Diaz an, dass bisherige Umfragen eine Ablehnung und Unzufriedenheit mit den Maßnahmen männlich geführter Regierungen nahelegen. Smith geht auf die Rolle weiblicher Personen bei COVID-19-Pressebriefings im Vereinigten Königreich ein [46]. Die Briefings sind männlich dominiert, nahezu 
die Hälfte aller Briefings wurde ausschließlich von Männern abgehalten. Bringt man diese Erkenntnis mit den Ausführungen von Piazza und Diaz zusammen, so wird die Problematik dieser Ungleichheit nochmals deutlicher. Obwohl es gute Gründe zur Annahme gibt, dass die Präsenz weiblicher Akteurinnen zu mehr Verständnis für Infektionsschutzmaßnahmen führen kann, werden weibliche Akteurinnen selten bis nicht repräsentiert und auch nicht zu Rat gezogen.

\subsection{Berichtete Reaktionen und Verhaltensweisen einzelner Gruppen}

In diesem Abschnitt werden nur exemplarisch einzelne Studien vorgestellt, welche gruppenspezifische Verhaltensweisen beziehungsweise die Auswirkungen der Medienberichterstattung auf die Gruppen untersucht haben. Dabei wird vor allem auf zwei Gruppen eingegangen: den Finanzmarkt und die Tourismusbranche. Anschließend wird der generelle Einfluss der Medienberichterstattung und des Rezeptionsverhaltens auf das Verhalten der Rezipierenden diskutiert.

Haroon und Rizvi analysieren den Zusammenhang zwischen der COVID-19bezogenen Medienberichterstattung, konkret den verwendeten „Panikbotschaften“, und den wirtschaftlichen Auswirkungen an Aktien- und Finanzmärkten [47]. Sie fanden Schwankungen an diesen Märkten, die auf die panikgenerierende Medienberichterstattung zurückzuführen sein können. Zu diesen Schwankungen kommt es laut den Analysen besonders in denjenigen Wirtschaftssektoren, die in der öffentlichen Meinung und Wahrnehmung am stärksten von der Pandemie betroffen sind.

Da vor allem im Frühjahr 2020 Reisen stark eingeschränkt wurden - und im Winter 2020/21 auch wieder nur eingeschränkt möglich sind -, ist es offensichtlich, dass gerade der Tourismussektor von der Pandemie stark beeinflusst wird. Die Medienberichterstattung über die Pandemie hat einen Einfluss auf die touristischen Reisen, vor allem indem die Einstellungen von potenziellen Touristinnen und Touristen beeinflusst werden und somit eine Sensibilisierung stattfindet [32]. Diese Einstellungsänderung und die verstärkte Sensibilisierung können sich auf das Reiseverhalten auswirken, sodass beispielsweise keine Reisen in öffentlichkeitswirksam benannte Risikogebiete geplant oder angetreten werden. Diese Verhaltensänderungen im Hinblick auf Reisen sind auch unmittelbar in unserer Umgebung auszumachen und mit der Medienberichterstattung in Deutschland in Einklang zu bringen. Im DACHRaum (Deutschland, Österreich, Schweiz) beobachten Neuburger und Egger im Frühjahr 2020 nicht nur eine geschärfte und gesteigerte Risikowahrnehmung gegenüber COVID-19, sondern auch eine gesteigerte Risikowahrnehmung gegenüber Reisen im Allgemeinen [48]. So änderte sich in diesem Gebiet auch das Reiseverhalten.

Abschließend soll noch ein kurzer Einblick in das Zusammenspiel von Medienberichterstattung und Rezeptionsverhalten der Leserschaft gegeben werden, der zur Diskussion anregen kann. In den USA wiesen Zhao et al. nach, dass das Mediennutzungsverhalten einen bedeutenden Einfluss auf das Präventionsverhalten haben kann [49]. Personen, die tendenziell ein höheres Vertrauen in die „Yellow Press“, also die Boulevardpresse haben, und Medien aus diesem Spektrum häufiger rezipieren, zeigen ein geringer ausgeprägtes Präventionsverhalten und neigen zu einem riskanteren Verhalten als Personen, die der Qualitätspresse ein höheres Vertrauen 
zusprechen und diese bevorzugt rezipieren. Da dies hier nur für die USA untersucht wurde, wären anknüpfende Untersuchungen auch im deutschsprachigen Raum und der hiesigen Medienlandschaft eine sinnvolle und wichtige Ergänzung, um das Akzeptanzverhalten in der Bevölkerung besser verstehen zu können.

\subsection{Berichtete Beachtung und Bewertung wissenschaftlicher Evidenz}

Evidenz gilt als Beleg für verwendete Hypothesen und Theorien. Insbesondere methodologische Kriterien sind hierbei wichtig; es geht um verwendete Methodendesigns, Erhebungsverfahren sowie Auswertungsstrategien und ihren Qualitätsstandards [50]. ${ }^{4}$ Wissenschaftliche sowie statistische Evidenz mit ihren Implikationen ist für den gesellschaftlichen Konsens relevant [51].

Um in der bekannten hierarchisierten Evidenzklassifizierung [55] ganz oben zu beginnen, ließe sich ermitteln,

- ob und inwieweit Ergebnisse von Systematic Reviews oder von Meta-Analysen oder von randomisierten Kontrollgruppenstudien als solche von den Medien berichtet und bewertet werden.

- ob in der Medienberichterstattung unsystematische Zusammenfassungen randomisierter Kontrollgruppenstudien, einzelne derartiger Studien oder Längsschnittanalysen vorkommen und wie sie behandelt und erörtert werden.

- ob zusammenfassende Übersichtsarbeiten zu ähnlichen Effekten aus unterschiedlichen medizinischen Perspektiven sowie über ähnliche Ergebnisse etwa aus ähnlichen medizinischen Ansätzen medial dargestellt und bewertet werden.

- inwiefern Einzelfallstudien aus Forschung und Klinikkontext zur Sprache kommen und in Medien erörtert beziehungsweise journalistisch eingeordnet werden.

Bisher sind nur wenige Studien erschienen, in denen der mediale Umgang mit der Evidenzproblematik überhaupt und eher allgemein angesprochen wird.

Konstatiert wird, dass in der COVID-19-Pandemie das öffentliche Bild von Wissen und Fachwissen und einer evidenzbasierten Politik aktueller geworden ist: „A standard practice of crisis communication, frequent live press conferences, has provided a powerful image of evidence based policy“" [56].

Zdravkovic et al. haben zur wissenschaftlichen Qualität der publizierten COVID19-bezogenen Inhalte in medizinischen Journals geforscht [57]. Dabei wurde die Stärke der Evidenz dieser publizierten Inhalte mit der Evidenzstärke der parallel veröffentlichten medizinischen Beiträge in den untersuchten Journals verglichen. Die Studie impliziert, dass die Beiträge ohne COVID-19-Bezug im Untersuchungszeitraum (März bis April 2020) und somit zu Beginn der Pandemie grundsätzlich eine höhere Evidenz aufweisen. Dies verdeutlicht, wie vorsichtig Ergebnisse interpretiert werden müssen und wie unsicher der Umgang mit neuen wissenschaftlichen Daten

4 Die seit Ende der 1990er Jahre v. a. von der DFG ausgehende Diskussion über Grundlagen der evidenzbasierten Medizin (EbM) wurden zunehmend öffentlich geführt [vgl. 20, 50, 52, 53]. Im Kontext des DFG-SPP 1409 „Wissenschaft und Öffentlichkeit“ wurde gefragt: (wie) wird wissenschaftliche Evidenz journalistisch dargestellt [vgl. 10, 11, 18, 20, 54]. 
ist. So wird die Bedeutung der Wissenschaftskommunikation und ebenso des Wissenschaftsjournalismus abermals verdeutlicht, um diese Ergebnisse auch für Laien nachvollziehbar einzuordnen und ein Grundverständnis für die wissenschaftliche Arbeitsweise und bestehende Unsicherheiten zu schaffen.

Hinzu kommt gerade angesichts des Ursprungs von COVID-19 in China die Frage, die immer wieder gerade von Journalistinnen und Journalisten diskutiert wird, ob und wie unterschiedliche Demokratie- und Gesellschaftssysteme medial mit der Unsicherheit wissenschaftlicher Erkenntnisse umgehen. Und ob sie dabei wichtiges und evidentes wissenschaftliches Wissen ${ }^{5}$ in der Berichterstattung ignorieren und übersehen [59].

Gerade die sozialen Medien erwiesen sich vor allem zu Beginn der Pandemie als besonders dynamisch [vgl. 34], um wissenschaftliche Informationen zwischen Wissenschaftlerinnen und Wissenschaftlern (Expertinnen und Experten), Regierungen (politische Entscheidungsträgerinnen und Entscheidungsträger), Massenmedien (Journalistinnen und Journalisten) und Bürgerinnen und Bürgern (Nichtfachleute) zu vermitteln und auszutauschen. Die bisher übliche wissenschaftliche Konsensbildung über empirisch gewonnene Fakten, logische Argumente und Theoriebildung verliert für Politik und Medien an Relevanz. Stattdessen geht es zunehmend auch um Alltagswissen, Episoden, verschiedene Emotionen und vermutete Trends. Sie verbreiten sich ungeprüft in Echtzeit in der Öffentlichkeit als vermeintlich wissenschaftliche Aussagen - begünstigt durch bestimmte Algorithmen [vgl. 60].

Als hilfreich können sich dabei bestimmte wissensbasierte Dienste und Plattformen erweisen, Entscheidungen in einer evidenzbasierten Politikgestaltung zumindest zu unterstützen und diese zu beschleunigen [vgl. 61, 62].

Zugleich werden aber auch Risiken beschleunigter und schneller PreprintPublikationen wissenschaftlich nicht gesicherter, nicht geprüfter oder nicht aktualisierter wissenschaftlicher Daten deutlich. Gerade solche Publikationen werden häufig sehr schnell von den Medien aufgegriffen und öffentlich verbreitet. Interessierte Bürgerinnen und Bürger, die Presse sowie die Öffentlichkeit können oft weder leicht noch unmittelbar die Preprint-Version von dem peer-reviewten Text unterscheiden. [vgl. 63] Das hat auch Implikationen für die Verbreitung von Informationen in sozialen Netzwerken, allerdings differenzierter als vermutet: „Research shows that, in social media, falsehood is shared far more than evidencebased information (...), false information is tweeted more but retweeted less than science-based evidence or fact-checking tweets, while science-based evidence and factchecking tweets capture more engagement than mere facts. " [64]

\subsection{Berichtete Behandlung von Desinformationen}

Im Kontext der laufenden COVID-19-Pandemie berichten vor allem journalistisch ausgerichtete Medien auch über verschiedene Formen von Desinformation zur Pandemie, ihre möglichen Wirkungen und Folgen. In der sozialpsychologischen

5 Etwa zur politischen Legitimation in China, aber auch in den USA durch Präsident Trump [58]. 
Forschung sind zugrunde liegende intergruppale und motivationale Prozesse für Desinformation schon länger erforscht worden [65]. Unter Stichwörtern wie „Verantwortung der Wissenschaft" wird entsprechend debattiert [vgl. 17, 66, 67].

Desinformationen werden - wie viele andere soziale Phänomene auch durch Medien weniger verursacht, als vielmehr aktualisiert, akzentuiert und dramatisiert, mit der Folge, dass sich krisenhafte Prozesse verstärken können [68, 69]. Die Kommunikationsforschung ist durch empirische Erfahrungen mit früheren Pandemien prädestiniert, Prozesse und Wirkungen der Desinformation empirisch und systematisch [27, 62, 71-73] zu analysieren. ${ }^{6}$

Eine Definitionsanalyse zeigt: „Aktuelle Desinformation kann (...) definiert werden als Kommunikation wissentlich und empirisch falscher Informationen $\mathrm{zu}$ neuen und relevanten Sachverhalten mit dem Anspruch auf Wahrheit" [74]. ${ }^{7}$

Erste Studien in Bezug auf Corona im Social Web (Facebook und Twitter sowie in Messenger-Diensten wie Telegram) zeigen solche aktuellen Desinformationen [vgl. 62, 73]. Sie beziehen sich auf Fragen unter anderem nach dem Ursprung des COVID-19-Virus, die Notwendigkeit von Hygieneregeln, möglichen pseudowissenschaftlichen Therapien und aktuell auch auf Impfstoffe und Impfwirkungen [27, 28]. Brennen et al. [75] finden in einer quantitativen Inhaltsanalyse vor allem Desinformationen $\mathrm{zu}$ Entscheidungen und Richtlinien, aber auch zur Kompetenz und Legitimität von staatlichen und internationalen Behörden, beispielsweise der WHO oder der Vereinten Nationen. Darüber hinaus hat eine qualitative Inhaltsanalyse Beispiele für desinformative visuelle Darstellungen untersucht [76]. Analysen von Social Media in Australien zeigen, wie eine kleine Anzahl von Aktivistinnen und Aktivisten Ad-hoc-Communities auf Twitter mobilisieren und regierungsfeindliche HashtagKampagnen starten können - zum Teil gemeinsam mit potenziell sympathisierenden Journalistinnen, Journalisten, Politikerinnen, Politikern sowie hochaktiven Meinungsführerinnen, Meinungsführern und ihren Anhängern, die Desinformationen, kritische Meinungen und Rhetoriken verbreiten [77].

Eine Studie, die auf breiter methodologischer und empirischer Basis entstanden ist, hat ein komplexes Einfluss-Modell zu Aktivitäten von Cyber-Crime, bezogen auf COVID-19, entwickelt. Analysiert werden aus einschlägigen Datenbanken ausgewählte Betrugsfälle inklusive Text, Bild und Fotos, spezifische Situationsfaktoren, Angriffsmethoden, soziale Netzwerke und mögliche Opfer [78].

Experimentelle Befunde zeigen zudem, dass sich unbewusstes Verhalten von Personen signifikant verändern kann, die einer Desinformation nur kurz ausgesetzt waren. Dass gefälschte Nachrichten verwendet werden können, um politisches Verhalten verdeckt zu ändern, lässt sich nicht ausschließen [79].

\footnotetext{
6 Aus der Film- und Theaterwissenschaft kommen erste interessante Bild-Text-Analysen und entsprechende diskursanalytische Überlegungen zur COVID-19-Pandemie [vgl. 70, S. 3 ff., $11 \mathrm{ff} ., 18 \mathrm{ff}$.].

7 Die eher absichtslos erstellten Falschinformationen über das Sars-CoV-2-Virus motiviert viele Bürgerinnen und Bürger tendenziell dazu weiter nach Informationen zu suchen [62]. Indes wissentlich eingesetzte Desinformationen [71] führen eher dazu, weitere Informationen zu meiden, diese auch nicht zu suchen und offizielle Regeln zu ignorieren.
} 


\subsection{Kurze Schlussbemerkungen}

Die Vermittlerrolle des Journalismus und des Wissenschaftsjournalismus, vor allem seine Zusammenarbeit mit Expertinnen und Experten, bleibt notwendig und wichtig. Hier sind Journalistinnen und Journalisten nicht nur in einer bloßen Vermittlerrolle tätig. Sondern sie sind auch eine Art Übersetzer, um die Öffentlichkeit mit adäquat aufbereiteten und selektierten wissenschaftlichen Informationen versorgen zu können. Wichtig ist diese selektive Leistung gerade vor dem Hintergrund der neben der Pandemie laufenden „Infodemie“, also einem öffentlichen Überangebot an Informationen. Dieses kann von den rezipierenden Laien kaum beherrscht und reflektiert eingeordnet werden. Gerade weil in Pandemiesituationen mit explizit journalistischer Kommunikation der Erfolg gerade von politischen oder juristischen Maßnahmen steht oder fällt. Dies war in westlichen Demokratien zunehmend selbstverständlich geworden. Allerdings lässt insbesondere die durchgreifende Kommerzialisierung der Medien seit den 1880er Jahren diese Einsicht fragiler werden [72].

Die öffentliche Nachfrage nach sozialwissenschaftlichem Wissen ist gerade in der momentanen Pandemie gestiegen. Denn hier ist nicht allein der direkte Arzt-Patienten-Kontakt wesentlich. Vielmehr ist die breite fach- und sachgerechte Kommunikation entscheidend, um öffentlich aufzuklären. Sie ergänzt die medizinische Behandlung der Erkrankten im klinischen Kontext. Der sozialwissenschaftliche Fokus auf wichtige (außerklinischen) Maßnahmen, in welchen eine Interdisziplinarität unabdingbar sind, sollte nicht nur zur Kenntnis genommen, sondern auch kritisch wertgeschätzt werden.

\section{Literatur}

1. https://www.spiegel.de/backstage/jahresrueckblick-2020-was-uns-beim-spiegel-dieses-jahrbewegt-hat-a-c4b032e9-bd41-420e-89c4-ecba39bf70a7. Zugegriffen: 20. Jan. 2021

2. Habermas, J.: „So viel Wissen über unser Nichtwissen gab es noch nie“. Frankfurter Rundschau (10.04.2020). https://www.fr.de/kultur/gesellschaft/juergen-habermas-coronaviruskrise-COVID19-interview-13642491.html. Zugegriffen: 20. Jan. 2021

3. Rosa, H.: Pfadabhängigkeit, Bifurkationspunkte und die Rolle der Soziologie. Ein soziologischer Deutungsversuch der Corona-Krise. Berliner J. Soziol. 30, 191-213 (2020)

4. Neuendorf, K.: The Content Analysis Guidebook. Sage, Thousand Oaks (2017)

5. Merten, K., Ruhrmann, G.: Die Entwicklung der inhaltsanalytischen Methode. Kölner Z. Soziol. Sozialpsychol. 34(4), 699 ff. (1982)

6. Früh, W., Früh, H.: Empirische Methoden in den Sozialwissenschaften und die Rolle der Inhaltsanalyse. Eine Analyse deutscher und internationaler Fachzeitschriften. In: Wirth, W., Sommer, K., Wettstein, M., Matthes, J. (Hrsg.) Qualitätskriterien in der Inhaltsanalyse 39ff. Herbert von Halem Verlag, Köln (2015)

7. Krippendorff, K.: Content Analysis. An Introduction to its Methodology, 4. Aufl., S. 188. Sage, Thousand Oaks (2019)

8. Guenther, L., Ruhrmann, G., Milde, J.: Pandemie: Wahrnehmung der gesundheitlichen Risiken durch die Bevölkerung und Konsequenzen für die Risiko- und Krisenkommunikation. Forschungsforum Öffentliche Sicherheit, Schriftenreihe Sicherheit Nr. 7, Berlin (2011) 
9. Sell, T.K., Hosangadi, D., Trotochaud, M.: Misinformation and the US Ebola communication crisis: Analyzing the veracity and content of social media messages related to a fear-inducing infectious disease outbreak. BMC Public Health 20, 550 (2020)

10. Guenther, L., Ruhrmann, G.: Scientific evidence and mass media: Investigating the journalistic intention to represent scientific uncertainty. Public Underst. Sci. 25(8), 927-943 (2016)

11. Maier, M., Milde, J., Post, S., Guenther, L., Ruhrmann, G., Barkela, B.: Communicating scientific evidence: Scientists', journalists' and audience expectations and evaluations regarding the representation of scientific uncertainty. Communications: Eur. J. Commun. Res. 41(3), 239-264 (2016)

12. Ruhrmann, G., Guenther, L.: Medizin- und Gesundheitsjournalismus. In: Rossmann, C., Hastall, M.R. (Hrsg.) Handbuch der Gesundheitskommunikation. Kommunikationswissenschaftliche Perspektiven, S. 69-79. Springer, Wiesbaden (2019)

13. Ruhrmann, G., Guenther, L.: Katastrophen- und Risikokommunikation. In: Bonfadelli, H., Fähnrich, B., Lüthje, C., Milde, J., Rhomberg, M., Schäfer, M.S. (Hrsg.) Forschungsfeld Wissenschaftskommunikation, S. 297-314. Springer VS, Wiesbaden (2017)

14. Ruhrmann, G., Daube, D.: Wissenschaftskommunikation in der Pandemie. Qualität und Perspektiven. G + G Wissenschaft 20(4), 7-14 (2020)

15. Daube, D., Ruhrmann, G.: Einführung zur Qualität von Medizinjournalismus und erste Einschätzungen zur COVID-19-Berichterstattung. Bundesgesundheitsblatt 64(1), 3-12 (2021)

16. Kessler, S.H., Guenther, L., Ruhrmann, G.: Die Darstellung epistemologischer Dimensionen von evidenzbasiertem Wissen in TV-Wissenschaftsmagazinen. Ein Lehrstück für die Bildungsforschung. Z. Erziehungswissenschaft 17(4), 119-139, Sonderheft 27/29014 (2014)

17. Leopoldina, acatech, Union der forschenden Akademien der Wissenschaften: Social Media und digitale Wissenschaftskommunikation. Analyse und Empfehlungen zum Umgang mit Chancen und Risiken in der Demokratie. Leopoldina, Halle (2017)

18. Maier, M., Guenther, L., Ruhrmann, G., Barkela, B., Milde, J.: Kommunikation ungesicherter wissenschaftlicher Evidenz - Herausforderungen für Wissenschaftler, Journalisten und Publikum. In: Janich, N., Rhein, L. (Hrsg.) Unsicherheit als Herausforderung in der Wissenschaft. Reflexionen aus Natur-. Sozial- und Geisteswissenschafte, S. 93-112. Lang, Berlin (2018)

19. Ruhrmann, G.: Kommunikation der Pandemie. Lichtgedanken. Forschungsmagazin der FSU Jena (2020)

20. Ruhrmann, G., Kessler, S.H., Guenther, L. (Hrsg.): Wissenschaftskommunikation zwischen Risiko und (Un)Sicherheit. Herbert von Halem Verlag, Köln (2016)

21. Klemm, C., Das, E., Hartmann, T.: Swine flu and hype: A systematic review of media dramatization of the H1N1 influenza pandemic. J. Risk Res. 19(1), 1 (2016)

22. Lin, L., Savoia, E., Agboola, F., Viswanath, K.: What have we learned about communication inequalities during the H1N1 pandemic: a systematic review of the literature. BMC Public Health 14, 484 (2014)

23. Alamoodi, A. H., Zaidan, B. B., Zaidan, A. A., Albahri, O. S., Mohammed, K. I., Malik, R. Q., Almahdi, E. M., Chyad, M. A., Tareq, Z., Albahri, A. S., Hameed, H., \& Alaa, M. Sentiment analysis and its applications in fighting COVID-19 and infectious diseases: A systematic review. Expert Systems with Applications, 167, 114155 (2021)

24. Metcalfe, J., Riedlinger, M., Bauer, M. W., Chakraborty, A., Gascoigne, T., Guenther, L., Joubert, M., Kaseje, M., Herrera-Lima, S., Revuelta, G., Riise, J., \& Schiele, B. The COVID-19 mirror: Reflecting science-society relationships across 11 countries. Journal of Science Communication, 19(07), A05 (2020)

25. Daube, D., Ruhrmann, G.: Systematic Review der Medienberichterstattung über die COVID-19-Pandemie (Arbeitstitel), in Arbeit 
26. Ludolph, R., Schulz, P.J., Chen, L.: Investigating the effects of mass media exposure on the uptake of preventive measures by Hong Kong residents during the 2015 MERS outbreak: The mediating role of interpersonal communication and the perception of concern. J. Health Commun. 23(1), 1-8 (2018)

27. Afzal, W.: What we can learn from information flows about COVID-19: Implications for research and practice. Proc. Assoc. Inf. Sci. Technol. 57, 245 (2020)

28. Naem, S.B., Bhatti, R., Khan, A.: An exploration of how fake news is taking over social media and putting public health at risk. Health Info. Libr. J. (2020). https://doi.org/10.1111/ hir. 12320

29. Anwar, A., Malik, M., Raees, V., Anwar, A.: Role of mass media and public health communications in the COVID-19 pandemic. Cureus 12(9), e10435 (2020)

30. Ogbodo, J.N., Onwe, E.C., Chukwu, J., Nwasum, C.J., Nwakpu, E.S., Nwankwo, S.U., Nwamini, S., Elem, S., Iroabuchi Ogbaeja, N.: Communicating health crisis: A content analysis of global media framing of COVID-19. Health Promot. Perspect. 10(3), 257-269 (2020)

31. Winter, S., Rösner, L.: Krisenkommunikation im Gesundheitsbereich. In: Rossmann, C., Hastall, M.R. (Hrsg.) Handbuch der Gesundheitskommunikation. Kommunikationswissenschaftliche Perspektiven, S. 423-432. Springer, Wiesbaden (2019)

32. Chemli, S., Toanoglou, M., Valeri, M.: The impact of COVID-19 media coverage on tourist's awareness for future travelling. Curr. Issues Tour. 1-8 (2020) https://doi.org/10.108 0/13683500.2020.1846502

33. Kreps, S. E., Kriner, D. L.: Model uncertainty, political contestation, and public trust in science: Evidence from the COVID-19 pandemic. Sci. Adv. 6(43), eabd4563 (2020)

34. Battiston, P., Kashyap, R. Rotondi, V.: Reliance on scientists and experts during an epidemic: evidence from the COVID-19 outbreak in Italy. SSM - Population Health. https://doi.org/10.1016/j.ssmph.2020.100721 (2020)

35. Broer, I.: Rapid reaction: Ethnographic insights into the Science Media Center and its response to the COVID-19 outbreak. J. Sci. Commun. 19(5), A08 (2020)

36. Stollorz, V.: Herausforderungen für den Journalismus über Wissenschaft in der Coronapandemie - erste Beobachtungen zu einem Weltereignis. Bundesgesundheitsblatt 64(1), 70-76 (2021)

37. Wormer, H.: German media and coronavirus: Exceptional communication or just a catalyst for existing tendencies? Media Commun. 8(2), 467-470 (2020)

38. Pollett, S., Rivers, C.: Social media and the new world of scientific communication during the COVID-19 pandemic. Clin. Infect. Dis. 71(16), 2184-2186 (2020)

39. Murri, R., Segala, F. V., Del Vecchio, P., Cingolani, A., Taddei, E., Micheli, G., Fantoni, M., COVID II Columbus Group: Social media as a tool for scientific updating at the time of COVID pandemic: Results from a national survey in Italy. PLOS One 15(9), e0238414 (2020)

40. Nowakowska, J., Sobocińska, J., Lewicki, M., Lemańska, Ż., Rzymski, P.: When science goes viral: The research response during three months of the COVID-19 outbreak. Biomed. Pharmacother. 129, 110451 (2020)

41. King, A.: Fast news or fake news? The advantages and the pitfalls of rapid publication through pre-print servers during a pandemic. EMBO Rep. 21(6), 1-4 (2020)

42. Abbasi, K.: COVID-19: Politicisation, "corruption", and suppression of science. BMJ, m4425 (2020) https://doi.org/10.1136/bmj.m4425

43. Tejedor, S., Cervi, L., Tusa, F., Portales, M., Zabotina, M.: Information on the COVID-19 Pandemic in daily newspapers' front pages: Case study of Spain and Italy. Int. J. Environ. Res. Public Health 17(17), 6330 (2020) 
44. Lasco, G.: Medical populism and the COVID-19 pandemic. Glob. Public Health 15(10), 1417-1429 (2020)

45. Piazza, K. S., Diaz, G.: Light in the midst of chaos: COVID-19 and female political representation. World Dev. 136, 105125 (2020)

46. Smith, J. C.: Where are the women? Descriptive representation and COVID-19 in U.K. Daily Press Briefings. Polit. Gender 16(4), 1-10 (2020)

47. Haroon, O., Rizvi, S. A. R: COVID-19: Media coverage and financial markets behavior-A sectoral inquiry. J. Behav. Exp. Finance 27, 100343 (2020)

48. Neuburger, L., \& Egger, R. Travel risk perception and travel behaviour during the COVID19 pandemic 2020: A case study of the DACH region. Current Issues in Tourism, 24(7), 1003-1016 (2021)

49. Zhao, E., Wu, Q., Crimmins, E. M., Ailshire, J. A.: Media trust and infection mitigating behaviours during the COVID-19 pandemic in the USA. BMJ Global Health 5(10), e003323 (2020)

50. Bromme, R. Prenzel, M., Jäger, M.: Empirische Bildungsforschung und evidenzbasierte Bildungspolitik. Eine Analyse von Anforderungen an die Darstellung, Interpretation und Rezeption empirischer Befunde. Z. Erziehungswissenschaft 27, Sonderheft, 3-54 (2014)

51. Leopoldina: Ad-hoc-Stellungnahmen zur Coronavirus-Pandemie (1-7). Stand: 08. Dezember 2020. Leopoldina, Halle/S. (2020). https://www.leopoldina.org/publikationen/ detailansicht/publication/leopoldina-stellungnahmen-zur-coronavirus-pandemie-2020/

52. Bromme, R., Goldman, S.E.: The public's bounded understanding of science. Educ. Psychol. 49(2), 59-69 (=Issue 2: Understanding the Public Understanding of Science: Psychological Approaches) (2014)

53. Ruhrmann, G.: Das öffentliche Bild von Biotechnologie und die Kommunikation von Evidenz. In: Weitze, M.-D., Pühler, A., Heckl, W.M., Müller-Röber, B., Renn, O., Weingart, P., Wess, G. (Hrsg.) Biotechnologie-Kommunikation. Kontroversen, Analysen, Aktivitäten, S. 287-302. Springer Vieweg, Heidelberg (2012)

54. Ruhrmann, G., Maier, M., Milde, J: Kommunikation wissenschaftlicher Evidenz von Zukunftstechnologien und ihre Wirkung auf Medienrezipienten. In: Kienhues, D. \& Bromme, R. (Hrsg.): Science and the Public. Das Verständnis fragiler und konfligierender Evidenz. Projekte, Forschungsthemen und Ergebnisse aus dem DFG-Schwerpunktprogramm 1409, 40-41 (2015)

55. Blümle, A., Meerpohl, J.J., Wolff, R., Antes, G.: Evidenzbasierte Medizin und systematische Übersichtsarbeiten. Die Rolle der Cochrane Collaboration. MKG-Chirurg 2009(2), 86-92 (2009)

56. Kunelius, R.: On the overlap of systemic events: COVID-19, climate, and journalism. Social Media + Society 6(3) 1-4, S. 2 (2020)

57. Zdravkovic, M., Berger-Estilita, J., Zdravkovic, B., Berger, D.: Scientific quality of COVID-19 and SARS CoV-2 publications in the highest impact medical journals during the early phase of the pandemic: A case control study. PLoS ONE 15(11), e0241826 (2020)

58. Altheide, D.: Pandemic in the time of Trump: Digital media logic and deadly politics (Editors invitation). Symb. Interact. 43(3), 514-540 (2020)

59. Zhang, Y., Xu, F.: Ignorance, orientalism and sinophobia in knowledge production on COVID-19. Tijdschr. Econ. Soc. Geogr. 111(3), 211-223 (2020)

60. van Dijck, J., Alinead, D.: Social media and trust in scientific expertise: Debating the COVID-19 pandemic in The Netherlands. Social Media + Society, 1-11 (2020)

61. El-Jardali, F., Bou-Karroum, L., Fadlallah, R.: Amplifying the role of knowledgetranslation platforms in the COVID-19 pandemic response. Health Res. Policy Syst. 18, 58 (2020) 
62. LfM NRW Landesanstalt für Medien Nordrhein- Westfalen NRW (Hrsg.): Informationslage und Meinungsbilder zu COVID-19: Auf der Suche nach Verlässlichkeit. Forschungsschwerpunkt Informationsintermediäre - Spezial, Ausgabe 6. Juli 2020. LfM-NRW, Düsseldorf (2020)

63. Bagdasarian, N., Cross, G.B., Fisher, D.: Rapid publications risk the integrity of science in the era of COVID-19. BMC Med. 18, 192 (2020)

64. Pulido, C.M., Villarejo-Carballido, B., Redondo-Sama, G., Gómez, A.: COVID-19 infodemic: More retweets for science-based information on coronavirus than for false information. Int. Sociol. 35(4) 377-392, S. 377 (2020)

65. Clark, C.J., Winegard, B.W.: Tribalism in war and peace: The nature and evolution of ideological epistemology and its significance for modern social science. Psychol. Inq. 31(1), 1-22 (2020)

66. Guenther, L., Ruhrmann, G., Weigelt, N., Zaremba, M. C.; The Newsworthiness of the "March for science" in Germany: Comparing news factors in journalistic media and on Twitter. J. Sci. Commun. (2021). Zugegriffen: 7. Dez. 2020

67. Guenther, L., Ruhrmann, G., Weigelt, N., Zaremba, M.C.: Facts, opinions, and scientific memes: Reflections of and recommendations for the March for Science in Germany. Sociol. Forum 34(4), 999-1008 (2019)

68. Chasdi, R. J., \& Ronis, S. R. A Study in Complexity: Unintended Consequences of Multiple Stakeholders in the U.S. Presidential Election Process. The International Journal of Intelligence, Security, and Public Affairs, 23(1), 48-62 (2021)

69. Theocharis, Y., \& Jungherr, A. Computational Social Science and the Study of Political Communication. Political Communication, 38(1-2), 1-22 (2021)

70. Pulos, R.: COVID-19 crisis memes, rhetorical arena theory and multimodality'. J. Sci. Commun. 19(07), A01 (2020)

71. Jungherr, A: Desinformation. Konzepte, Identifikation, Reichweite und Effekte. In: Landesanstalt für Medien Nordrhein- Westfalen NRW (Hrsg.): Was ist Desinformation Betrachtungen aus sechs wissenschaftlichen Perspektiven. LfM-NRW, Düsseldorf 23-30 (2020)

72. LfM NRW Landesanstalt für Medien Nordrhein-Westfalen NRW (Hrsg.): Was ist Desinformation. Betrachtungen aus sechs wissenschaftlichen Perspektiven. Lfm-NRW, Düsseldorf (2020)

73. LfM NRW Landesanstalt für Medien Nordrhein- Westfalen NRW (Hrsg.): Unsichtbare Stimmen - Ausschluss und Rückzug aus Online-Diskursen. Forschungsmonitor Informationsintermediäre Ausgabe 7: Dezember 2020 (2020)

74. Kohring, M, Zimmermann, F.: Aktuelle Desinformation. Definition, Konsequenzen, Gegenmaßnahmen. In: LfM NRW Landesanstalt für Medien Nordrhein- Westfalen NRW (Hrsg.): Was ist Desinformation? Betrachtungen aus sechs wissenschaftlichen Perspektiven, S. 15-22. LfM-NRW, S. 19 Düsseldorf (2020)

75. Brennen, J.S., Simon, F.M., Howard, P.N., Nielsen, R.K.: Types, sources, and claims of COVID-19 misinformation. RISJ Factsheet. Reuters Institute for the Study of Journalism (2020)

76. Brennen, J.S., Simon, F.M., Nielsen, R.K.: Beyond (Mis)Representation: Visuals in COVID-19 misinformation. Int. J. Press/Polit. 26(1), 277-299 (2020)

77. Graham, T., Bruns, A., Angus, D., Hurcombe, E., Hames, S.: Stand With Dan versus \#DictatorDan: the polarised dynamics of Twitter discussions about Victoria's COVID-19 restrictions. Media Int. Australia (2020)

78. Naidoo, R.: A multi-level influence model of COVID-19 themed cybercrime. Eur. J. Inf. Syst. 29(3), 306-321 (2020)

79. Bastick, Z. Would you notice if fake news changed your behavior? An experiment on the unconscious effects of disinformation. Computers in Human Behavior, 116, 106633 (2021) 


\section{Prof. Dr. Georg Ruhrmann}

(geb. 1955), studierte Philosophie, Molekularbiologie und Soziologie an den Universitäten Marburg und Bielefeld. 1997 Ruf an die TU Ilmenau (abgelehnt); 1998 Ruf an die Friedrich-Schiller-Universität Jena; 2003 Ruf an die Friedrich-Alexander Universität Erlangen/Nürnberg (abgelehnt). 2002 bis 2006 Projektleitungen im BMBF Projekt "Molekulare Medizin und Medien" sowie 2009 bis 2016 im DFGSchwerpunktprogramm 1409 „Wissenschaft und Öffentlichkeit“. Seit 2009 Mitglied der Kommission für Risikoforschung und -wahrnehmung beim Bundesinstitut für Risikobewertung (BfR). Seit 2019 Mitglied der Versammlung der Thüringer Landesmedienanstalt (TLM). Ruhrmann lehrt seit 2019 auch an der Hochschule für Recht und Wirtschaft Berlin (HWR) und ist seit 2020 Modulverantwortlicher im Studienprogramm "eHealth \& Communication" am Universitätsklinikum Jena (UKJ). Seit April 2021 Seniorprofessur für Kommunikationswissenschaft mit dem Schwerpunkt Digitalisierung und Öffentlichkeit an der Friedrich-Schiller-Universität Jena.

\section{Dominik Daube (M.A.)}

(geb. 1993), studierte im Bachelor Kommunikationswissenschaft und Staatswissenschaften - Rechtswissenschaft (B.A.) an der Universität Erfurt. Es folgte 2017 bis 2019 ein Master-Studium der Gesundheitskommunikation (M.A.) an der Universität Erfurt. Praktische Erfahrung sammelte Daube in der Unternehmenskommunikation eines Klinikums. Von 2019 bis 2021 war Daube Wissenschaftlicher Mitarbeiter in Forschung und Lehre am Lehrstuhl Grundlagen der medialen Kommunikation und der Medienwirkung am Institut für Kommunikationswissenschaft (IfKW) an der Friedrich-Schiller-Universität Jena. Seit April 2021 ist Daube Wissenschaftlicher Mitarbeiter in Forschung und Lehre an der Professur Kommunikationswissenschaft mit dem Schwerpunkt Digitalisierung und Öffentlichkeit am IfKW der Friedrich-SchillerUniversität Jena. Er ist seit 2020 beteiligt an der Vorbereitung des Studienprogramms eHealth \& Communication am Universitätsklinikum Jena (UKJ) und lehrt seit dem Sommersemester 2021 in diesem Studienprogramm.

Open Access Dieses Kapitel wird unter der Creative Commons Namensnennung - Nicht kommerziell - Keine Bearbeitung 4.0 International Lizenz (http://creativecommons.org/ licenses/by-nc-nd/4.0/deed.de) veröffentlicht, welche die nicht-kommerzielle Nutzung, Vervielfältigung, Verbreitung und Wiedergabe in jeglichem Medium und Format erlaubt, sofern Sie den/die ursprünglichen Autor(en) und die Quelle ordnungsgemäß nennen, einen Link zur Creative Commons Lizenz beifügen und angeben, ob Änderungen vorgenommen wurden. Die Lizenz gibt Ihnen nicht das Recht, bearbeitete oder sonst wie umgestaltete Fassungen dieses Werkes zu verbreiten oder öffentlich wiederzugeben.

Die in diesem Kapitel enthaltenen Bilder und sonstiges Drittmaterial unterliegen ebenfalls der genannten Creative Commons Lizenz, sofern sich aus der Abbildungslegende nichts anderes ergibt. Sofern das betreffende Material nicht unter der genannten Creative Commons Lizenz steht und die betreffende Handlung nicht nach gesetzlichen Vorschriften erlaubt ist, ist auch für die oben aufgeführten nicht-kommerziellen Weiterverwendungen des Materials die Einwilligung des jeweiligen Rechteinhabers einzuholen.

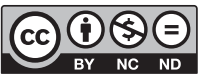

